\title{
Blood Glucose Control in Neonatal Intensive Care with Model-Based Controllers
}

\author{
Aaron J. Le Compte*, J. Geoffrey Chase*, Adrienne Lynn**, Chris E. Hann*, Geoffrey M. Shaw***, Jessica Lin* \\ * Department of Mechanical Engineering, University of Canterbury, Christchurch, New Zealand \\ ** Neonatal Department, Christchurch Women's Hospital, Christchurch, New Zealand \\ *** Department of Intensive Care, Christchurch Hospital, Christchurch, New Zealand
}

\begin{abstract}
Premature neonates often experience hyperglycaemia, which has been linked to increased mortality and worsened outcomes. Insulin therapy can assist in controlling blood glucose levels. However a reliable, robust control protocol is required to avoid hypoglycaemia and to meet nutrition goals. This study presents an adaptive, model-based predictive controller designed to incorporate the unique metabolic state and control parameters of the neonate. Controller performance was tested in virtual trials on a 25 patient retrospective cohort and 24-hour pilot clinical trials. The effects of measurement frequency and BG sensor error were also evaluated. Time in the $4-7 \mathrm{mmol} / \mathrm{L} \mathrm{BG}$ band was increased by $110 \%-145 \%$ compared to retrospective control for that cohort, with fewer hypoglycaemic measurements. Controllers were robust to BG sensor errors.
\end{abstract}

Keywords: Insulin sensitivity, control algorithms, physiological models, simulation, intensive care

\section{INTRODUCTION}

Premature infants commonly demonstrate poor glycaemic control. Up to $40 \%-80 \%$ of low birth weight infants experience hyperglycaemia during the neonatal period (Dweck and Cassady, 1974). Metabolic homeostasis in the preterm infant is often compromised by immaturity of control systems. Additionally, the metabolic response to stress is characterised by major changes in glucose metabolism (Ditzenberger et al., 1999). Increased secretion of counterregulatory hormones leads to a prominent rise in endogenously produced glucose and the rate of hepatic gluconeogenesis, as well as a reduction in insulin sensitivity. Inhibiting the physiological response to increased glycaemic levels are factors such as increased insulin resistance, absolute or relative insulin deficiency, and drug therapy.

Hyperglycaemia is not only a marker for severity of illness, it also has been linked to worsened outcomes, leading to an increased risk of further complications, such as sepsis, retinopathy of prematurity and mortality (Van den Berghe et al., 2001, Ertl et al., 2006). High rates of proteolysis are also common in low birth weight infants, reducing muscle mass and inhibiting growth (Agus et al., 2004).

Tight glucose control has been shown to reduce adult Intensive Care Unit (ICU) patient mortality by $45 \%$ (Van den Berghe et al., 2001). Prospective tight glucose control to a mortality and/or morbidity outcome has not yet been reported for the neonatal intensive care unit (NICU).

Hyperglycaemia in preterm neonates is often treated by glucose restriction and/or the use of insulin infusions. Insulin infusions are gaining popularity amongst neonatologists, where they were once avoided due to the risk of hypoglycaemia and possible neurological sequelae. Limited trials of insulin infusions have been reported (Kairamkonda, 2006). Positive outcomes of insulin infusion have been reduced proteolysis, improved glucose tolerance and improved weight gain. Negative reports of hypoglycaemia have also surfaced (Beardsall et al., 2008).

Blood glucose control for the neonate poses several challenges that differ from the adult critical care case. Blood volumes in preterm infants are relatively small. Thus, the number of blood glucose measurements must be optimised to a minimum useful number to conserve volume and restrict opening incubator doors, which may affect the neonate's hydration status. Endogenous energy substrates are very low in preterm infants at birth (Hume et al., 2005). Thus, these infants must be constantly fed to provide enough energy for basal requirements in addition to growth. In contrast, adults can tolerate periods of reduced caloric intake. Less saturation of the insulin-stimulated glucose uptake pathway has also been reported in neonates (Farrag et al., 1997) unlike in adults (Natali et al., 2000). Finally, also unlike the adult case, growth is a major goal of neonatal care. Thus, the anabolic effects of insulin are of relatively high importance (Agus et al., 2004).

Great inter-patient heterogeneity in response to glucose and insulin infusions is a well-reported hallmark of neonatal glucose metabolism making safe, adequate control difficult (Cowett and Farrag, 2004). Thus, knowledge of the metabolic state of the infant is vital for optimal, safe blood glucose control using insulin. Model-based methods can provide information about the patient's response to insulin based on serial blood glucose measurements, and insulin and nutrition data (Chase et al., 2006). This metabolic information can be combined with a controller utilising model predictions to achieve targeted blood glucose control. This approach has been validated in adult critical care studies (Chase et al., 2006). However, sudden changes in patient condition independent of metabolic state indicate limits on model-based 
controller actions are required to maximise safety and control performance.

Therefore, there are several design parameters that must be considered in developing a safe, effective and optimal neonatal glycaemic control algorithm. Virtual trials offer the opportunity to explore control strategies in simulation before pilot clinical trials (Chase et al., 2007). The controller must account for inter-patient variability, nutritional inputs and varying physiological condition. Hence, it must be adaptive and/or able to identify changes in patient dynamics, particularly with respect to insulin sensitivity. The protocol should minimise labour and comply with existing medical protocols on the treatment of neonatal hyperglycaemia to ensure the method developed could be readily implemented in a clinical environment. Finally, the controller must be robust to sensor errors.

\section{METHODS}

\subsection{System model}

The model is based on a clinically validated adult critical care glycaemic model, adapted to account for the main physiological differences in neonates.

$$
\begin{aligned}
\dot{G}= & -p_{G} \cdot G-S_{I} \cdot G \cdot \frac{Q}{1+\alpha_{G} Q} \\
& +\frac{P(t)+\left(P_{E N D} \cdot m_{\text {body }}\right)-\left(C N S \cdot m_{\text {brain }}\right)}{\left(V_{G, \text { frac }}(t) \cdot m_{\text {body }}\right)} \\
\dot{Q}= & -k Q+k I \\
\dot{I}= & -\frac{n I}{1+\alpha_{I} I}+\frac{u_{e x}(t)}{\left(V_{I, \text { frac }} \cdot m_{\text {body }}\right)}+e^{-\left(k_{l} u_{e x}(t)\right)} I_{B}
\end{aligned}
$$

Where $G(t)[\mathrm{mmol} / \mathrm{L}]$ is the total plasma glucose and $I(t)$ $[\mathrm{mU} / \mathrm{L}]$ is the plasma insulin, exogenous insulin input is represented by $u_{e x}(\mathrm{t})[\mathrm{mU} / \mathrm{min}]$ and basal endogenous insulin secretion $I_{B}[\mathrm{mU} / \mathrm{L} / \mathrm{min}]$, with $k_{I}$ representing the suppression of basal insulin secretion in the presence of exogenous insulin. The effect of previously infused insulin being utilised over time is represented by $Q(t)$ [mU/L], with $k[1 / \mathrm{min}]$ accounting for the effective life of insulin in the system. Body weight and brain weight are denoted by $m_{\text {body }}[\mathrm{kg}]$ and $m_{\text {brain }}[\mathrm{kg}]$ respectively. Patient endogenous glucose clearance and insulin sensitivity are $p_{G}[1 / \mathrm{min}]$ and $S_{I}$ [L/(mU.min)], respectively. The parameter $V_{I, \text { frac }}[\mathrm{L} / \mathrm{kg}]$ is the insulin distribution volume per kilogram body weight and $n$ [1/min] is the constant first order decay rate for insulin from plasma. Total plasma glucose input is denoted $P(t)$ [mmol $/ \mathrm{min}]$, endogenous glucose production is denoted by $P_{E N D}$ [mmol/kg/min] and $V_{G, f r a c}[\mathrm{~L} / \mathrm{kg}]$ represents the glucose distribution volume per kilogram of body weight. CNS [mmol $/ \mathrm{kg} / \mathrm{min}]$ represents non-insulin mediated glucose uptake by the central nervous system, as well as the liver, kidneys and red blood cells. Michaelis-Menten functions are used to model saturation, with $\alpha_{I}[\mathrm{~L} / \mathrm{mU}]$ used for the saturation of plasma insulin disappearance, and $\alpha_{G}[\mathrm{~L} / \mathrm{mU}]$ for the saturation of insulin-dependent glucose clearance.

Table 1 summarises the parameters values. In this study, $k, n$, $\alpha_{I}, \alpha_{G}, C N S, I_{B}, V_{I, f r a c}, p_{G}$ and $P_{E N D}$ are set to population values based on reported clinical neonate data. Prior clinical and model sensitivity studies (Hann et al., 2005) have shown this choice to be robust.

Table 1. Model parameter values

\begin{tabular}{|c|c|}
\hline Parameter & Value \\
\hline$k$ & $0.0086 \mathrm{~min}^{-1}$ \\
$n$ & $0.90 \mathrm{~min}^{-1}$ \\
$\alpha_{I}$ & $1.70 \times 10^{-3} \mathrm{~L} / \mathrm{mU}$ \\
$\alpha_{G}$ & $0 \mathrm{~L} / \mathrm{mU}$ \\
$C N S$ & $0.088 \mathrm{mmol} / \mathrm{kg} / \mathrm{min}$ \\
$m_{\text {brain }}$ & $0.14 * m_{\text {body }} \mathrm{kg}$ \\
$I_{B}$ & $12 \mathrm{mU} / \mathrm{L} / \mathrm{min}$ \\
$V_{I, \text { frac }}$ & $0.045 \mathrm{~L} / \mathrm{kg}$ \\
$V_{G, \text { frac }}$ & (Based on gestational age) $\mathrm{L} / \mathrm{kg}$ \\
$P_{E N D}$ & $0.02838 \mathrm{mmol} / \mathrm{kg} / \mathrm{min}$ \\
$p_{G}$ & $0.003 \mathrm{~min}^{-1}$ \\
\hline
\end{tabular}

The insulin sensitivity parameter, $S_{I}$, drives the dynamics of the blood glucose model and is assumed patient-specific and independent of exogenous insulin and nutrition administration. Once a patient-specific profile of timevarying insulin sensitivity is generated, it can be used to predict blood glucose concentration based on different insulin and nutrition control schemes. Such analyses are effectively in-silico or virtual trials (Chase et al., 2007).

\subsection{Controller development}

The clinical implementation procedure for the controller is shown in Fig. 1. The blood glucose history, together with insulin and nutrition history, are used to fit the patient's insulin sensitivity profile in real-time. This profile is then used by the controller to solve (1)-(3) to predict blood glucose concentration based on insulin and nutrition rates. Thus, the controller adapts to the current metabolic state of the neonate in real-time.

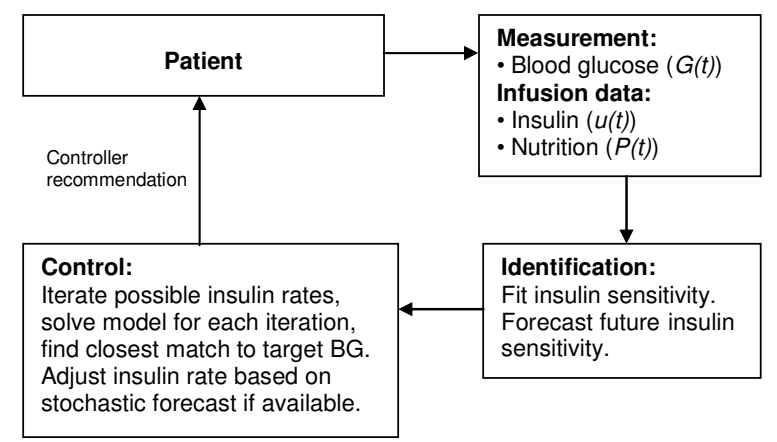

Fig. 1. Controller implementation schematic

The controller uses a bisection algorithm to determine the insulin infusion rate that will bring the BG closest to a target $\mathrm{BG}$, if the target is physiologically possible with safety. Thus, every BG measurement is followed by a controller intervention to alter the insulin infusion rate. The virtual trial procedure replaces the 'Patient' in Fig. 1 with a forward solution of the model using an insulin sensitivity profile generated from retrospective data, and adds sensor noise and other variations as required. 
Crucially, blood volumes in neonates are small (Cassady, 1966), which restricts the frequency of BG sampling, providing an additional challenge for model-based control in the neonatal setting. Thus, it is important to optimise the number of BG measurements required for control.

Controllers using $1-4$ hourly BG measurement and intervention intervals were examined and compared to retrospective hospital control and simulations using an insulin sliding scale as per standard care in Christchurch Women's Neonatal Department, shown in Table 2.

Table 2. Typical sliding scale for simulation.

\begin{tabular}{cc}
\hline Blood glucose & Insulin rate \\
$>20 \mathrm{mmol} / \mathrm{L}$ & $0.100 \mathrm{U} / \mathrm{kg} / \mathrm{hr}$ \\
$15-20 \mathrm{mmol} / \mathrm{L}$ & $0.075 \mathrm{U} / \mathrm{kg} / \mathrm{hr}$ \\
$10-15 \mathrm{mmol} / \mathrm{L}$ & $0.050 \mathrm{U} / \mathrm{kg} / \mathrm{hr}$ \\
$5-10 \mathrm{mmol} / \mathrm{L}$ & $0.025 \mathrm{U} / \mathrm{kg} / \mathrm{hr}$ \\
$<5 \mathrm{mmol} / \mathrm{L}$ & $\mathrm{STOP}$ \\
\hline
\end{tabular}

A BG measurement timing scheme based on current BG concentration was also tested. In particular, a high BG concentration carries little risk of hypoglycaemia and may thus require less frequent $\mathrm{BG}$ sampling compared to periods at lower concentrations. Hence, a BG-concentration derived measurement scheme was simulated where measurements were taken every 3 hours if $\mathrm{BG}>8 \mathrm{mmol} / \mathrm{L}$ and any decrease in $\mathrm{BG}$ since the last measurement was less than $2 \mathrm{mmol} / \mathrm{L} / \mathrm{hr}$. Measurements were taken 2-hourly if BG was within the 4-8 $\mathrm{mmol} / \mathrm{L}$ range, and hourly otherwise up to a limit of 12 measurements per day. Unless otherwise stated, all simulations were performed with uniformly distributed $7 \%$ measurement error (Chen et al., 2003).

The metabolic status of a critically ill neonatal patient can change rapidly. This change is reflected by sudden rises and drops in insulin sensitivity. Additionally, sudden changes in apparent insulin sensitivity may be caused by sensor noise and/or measurement error (Chase et al., 2007). Thus, a balance is required between the speed at which a controller reacts to correct blood glucose rises due to sudden changes in metabolic state, and the risk of running higher insulin infusion rates when a sudden rise in blood glucose resolves quickly, or was due to measurement error. A maximum insulin infusion rate of $0.5 \mathrm{U} / \mathrm{kg} / \mathrm{hr}$ and peak increase of 0.03 $\mathrm{U} / \mathrm{kg} / \mathrm{hr}$ per intervention were used in the model-based controller.

Finally, the effect of BG sensor error was explored in simulated trials by adjusting the amount of uniformly distributed noise added to simulated BG measurements. Values of $2 \%, 5 \%, 10 \%, 15 \%$ and $20 \%$, represent the range (and beyond) seen in clinical practice depending on the BG sensor/analyser used (Chen et al., 2003).

\subsection{Patient cohort}

Retrospective data for 25 episodes of insulin usage over 21 patients from the Christchurch Women's Neonatal Intensive Care Unit was used in the study. Ethics approval for the collection and publication of data was obtained from the Upper South Regional Ethics Committee. Median gestational age at birth was 26.6 weeks, and median birth weight was $0.845 \mathrm{~kg}$. Inclusion criteria were a period of treatment with insulin and at least six blood glucose measurements per day. Hourly-varying insulin sensitivity was fitted to each of the 25 patient profiles to generate a cohort of 25 'virtual patients' used for simulation.

Two 24-hour pilot clinical trials of the model-based controller are presented. The two subjects were born at $27.3 \mathrm{wks}$ and $25.4 \mathrm{wks}$ gestational age and birth weight 0.77 $\mathrm{kg}$ and $0.72 \mathrm{~kg}$ respectively. The trials were conducted at age 9 days and 2 days respectively.

\section{RESULTS}

Table 3 compares blood glucose performance metrics and insulin intake for clinical retrospective NICU control, simulated sliding scale, and targeted control. The blood glucose target is $6.0 \mathrm{mmol} / \mathrm{L}$ or a $15 \%$ reduction per hour from the current $\mathrm{BG}$ concentration, whichever is the greater value. The median BG for all model-based control cases is at, or close, to the target BG. The percentage of measurements within the $4-7 \mathrm{mmol} / \mathrm{L}$ and $4-8 \mathrm{mmol} / \mathrm{L}$ ranges are $[110 \%$ - $145 \%]$ and $[65 \%-87 \%]$ higher than retrospective hospital control, respectively. The sliding scale had higher median BG than either model-based control or retrospective results. A sample model fit to the data simulation of a sliding scale controller is presented in Fig. 2.

Table 3. Simulated control results and retrospective data.

\begin{tabular}{|c|c|c|c|}
\hline & \multicolumn{3}{|c|}{ Model-based controller } \\
\hline & $2 \mathrm{hr}$ & $3 \mathrm{hr}$ & $4 \mathrm{hr}$ \\
\hline Total measurements & 1771 & 1175 & 879 \\
\hline Median BG (mmol/L) & 6.0 & 6.0 & 6.1 \\
\hline $\mathrm{BG} \mathrm{IQR}^{\mathrm{a}}(\mathrm{mmol} / \mathrm{L})$ & $\begin{array}{c}{[5.4-} \\
6.8]\end{array}$ & $\begin{array}{c}{[5.3-} \\
7.0]\end{array}$ & $\begin{array}{c}{[5.2-} \\
7.2]\end{array}$ \\
\hline$\%$ within $4-7 \mathrm{mmol} / \mathrm{L}$ & $76 \%$ & $69 \%$ & $65 \%$ \\
\hline$<2.6 \mathrm{mmol} / \mathrm{L}$ & $0.45 \%$ & $0.60 \%$ & $0.91 \%$ \\
\hline \multirow[t]{3}{*}{ Insulin intake $(\mathrm{U} / \mathrm{kg} / \mathrm{hr})$} & 0.056 & 0.057 & 0.055 \\
\hline & \multicolumn{2}{|c|}{ Sliding scale } & \multirow{2}{*}{ Retro. } \\
\hline & $2 \mathrm{hr}$ & $4 \mathrm{hr}$ & \\
\hline Total measurements & 1771 & 879 & 1091 \\
\hline Median BG (mmol/L) & 8.6 & 8.5 & 8.0 \\
\hline $\mathrm{BG} \mathrm{IQR}^{\mathrm{a}}(\mathrm{mmol} / \mathrm{L})$ & $\begin{array}{l}{[6.9-} \\
10.5]\end{array}$ & $\begin{array}{l}{[6.9-} \\
10.8]\end{array}$ & $\begin{array}{c}{[6.3-} \\
9.9]\end{array}$ \\
\hline$\%$ within $4-7 \mathrm{mmol} / \mathrm{L}$ & $25 \%$ & $24 \%$ & $31 \%$ \\
\hline$<2.6 \mathrm{mmol} / \mathrm{L}$ & $0.34 \%$ & $0.57 \%$ & $0.73 \%$ \\
\hline Insulin intake (U/kg/hr) & 0.029 & 0.030 & 0.034 \\
\hline
\end{tabular}

${ }^{\mathrm{a}} \mathrm{IQR}$, Inter-Quartile Range.

The length of time between blood glucose measurements reduces the quality of model-based control, dropping from $76 \%$ to $65 \%$ of BG measurements within the $4-7 \mathrm{mmol} / \mathrm{L}$ band for 2-hour to 4-hour measurement intervals. The proportion of simulated measurements below $2.6 \mathrm{mmol} / \mathrm{L}$ is less than retrospective control for 2-hourly and 3-hourly measurements, but slightly greater for 4-hourly measurements. The IQR width for retrospective control was $3.6 \mathrm{mmol} / \mathrm{L}$, and $3.6-3.9 \mathrm{mmol} / \mathrm{L}$ for sliding scale control. It was $1.4-2.2 \mathrm{mmol} / \mathrm{L}$ for model-based control. Sliding scale control results were similar across measurement frequencies. 
Insulin usage across measurement frequencies was similar for model-based control, and 65\% - 74\% higher than hospital control. The highest proportion of measurements within the target band was for the 2-hour control.
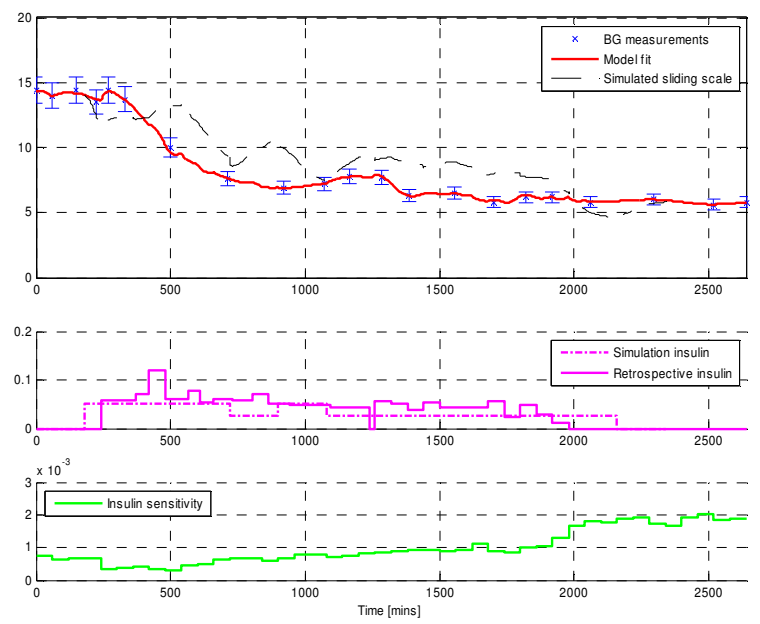

Fig. 2. Model fit through BG data and control simulation.

Fig. 3 shows the median patient cumulative distribution function (CDF) and 5\% - 95\% range of CDFs of the perpatient control results for the model-based controller with 2hour measurements, and retrospective data. The inter-patient variation in $\mathrm{BG}$ control with the model-based controller is far tighter than the retrospective control. Thus, the model-based controller better modulates insulin to account for each individual patient's glycaemic response.

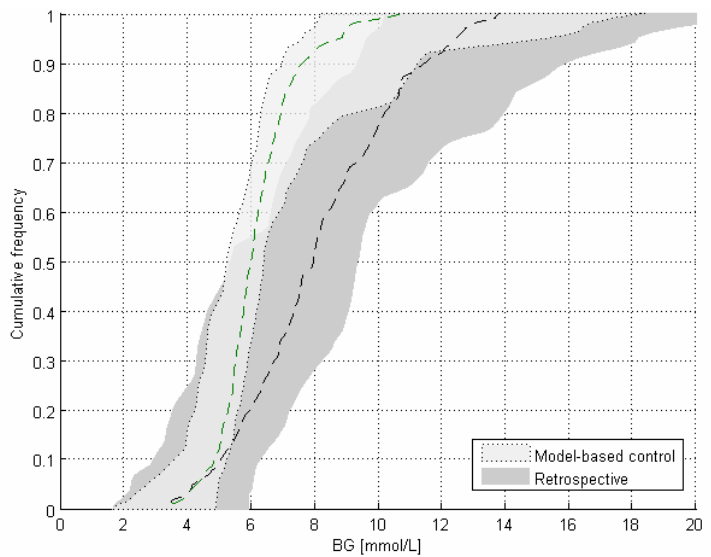

Fig. 3. Median and 5\%-95\% CDF of $\mathrm{BG}$ for simulated model-based control and retrospective data.

All model-based controller results are significantly different from the retrospective $B G$ measurement distribution $(\mathrm{p}<$ 0.05). It is important to note the curves presented in Fig. 3 are not symmetrical - the slope at lower BG ranges is steeper, thus BG results when not within the target range are skewed towards the upper BG range, as mild hyperglycaemia is considered safer than an increased risk of hypoglycaemia.

Fig. 4 compares the proportion of BG measurements within the target $4-7 \mathrm{mmol} / \mathrm{L}$ band for retrospective and modelbased control. Only one patient retrospectively had greater than $50 \%$ of $\mathrm{BG}$ measurements within the target band under hospital control. All patients had greater than $50 \%$ of BG measurements within the target band with model-based control. The $45^{\circ}$ line in Fig. 4 represents the line of nochange in performance. The distance from the line is a measure of the increase in BG measurements within the target band per-patient.

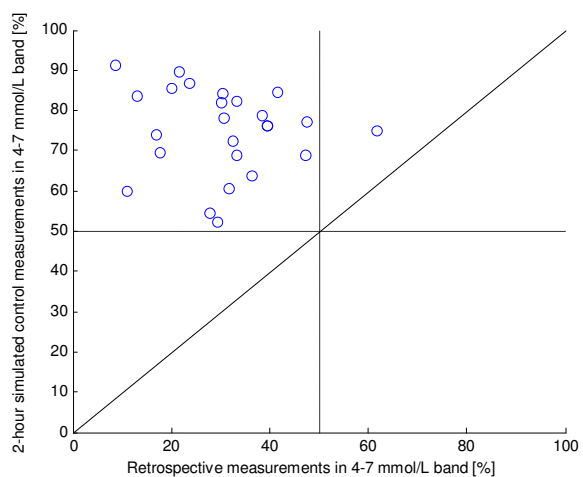

Fig. 4. Retrospective measurements within target BG range compared to model-based controller virtual trials.

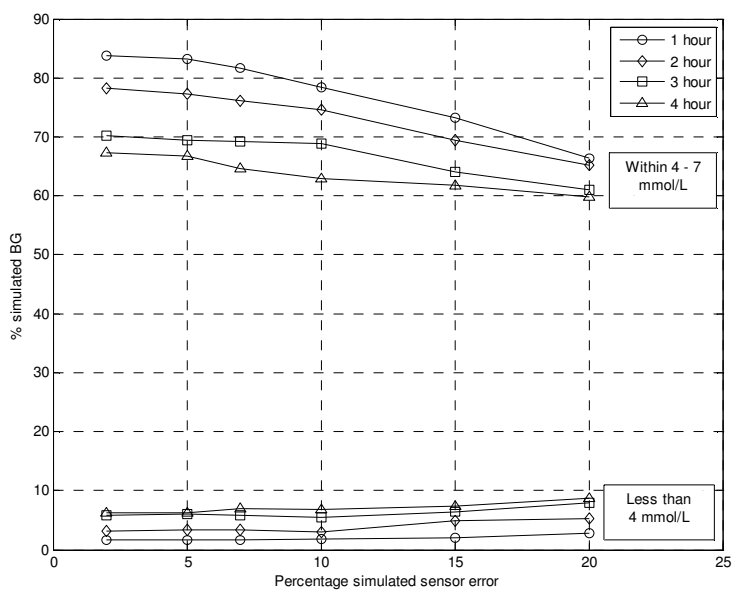

Fig. 5. Effect of simulated BG sensor error on BG control.

Fig. 5 compares glycaemic control performance with different measurement errors for different intervals. Most accurate control was achieved with the minimum measurement noise, as expected. Decreases in time in glycaemic control bands showed a similar pattern for all measurement frequencies.

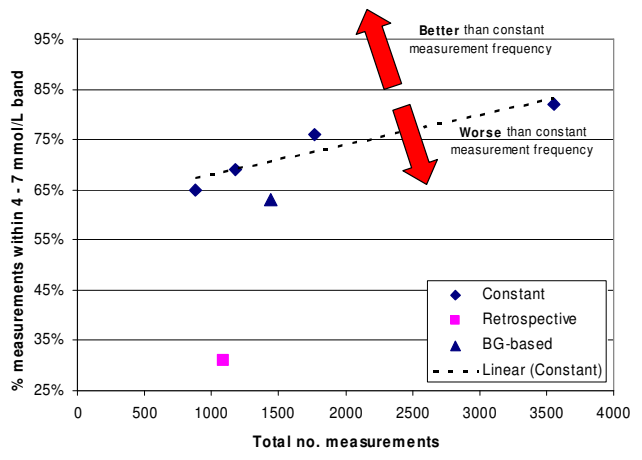

Fig. 6. Measurements within target $\mathrm{BG}$ range compared to number of measurements. 
The BG concentration-based variable measurement frequency scheme resulted in lower time in target BG band, higher median BG and wider BG inter-quartile range. This result is emphasised in Fig. 6, which compares the percentage of BG measurements with the target band against total simulated BG measurements for several BG measurement schemes. The retrospective and BG-concentration based measurement frequency results are below a linear line through the constant measurement frequency results. Thus, these measurement frequency schemes do not make a more optimal use of the measurements available compared to the clinically easier regular measurement frequency.

Fig. 7 and Fig. 8 present two 24-hour clinical control trials. In both cases blood glucose concentration is reduced in a controlled manner to the target $4-7 \mathrm{mmol} / \mathrm{L}$ band. BG prediction accuracy was $9.8 \%$ and $8.8 \%$ for Trials 1 and 2 respectively. Insulin sensitivity for Trial 2 was much lower than Trial 1, providing a broad indication of severity of illness and ability of insulin to maintain tight control. Up to $60 \%$ of dextrose calories in Trial 2 were administered via morphine and dobutamine solutions, in addition to parenteral nutrition. Controller BG concentration targets for Trial 2 aimed for a $15 \%$ reduction in glycaemia to achieve a steady, controlled descent in glucose levels to the target range.
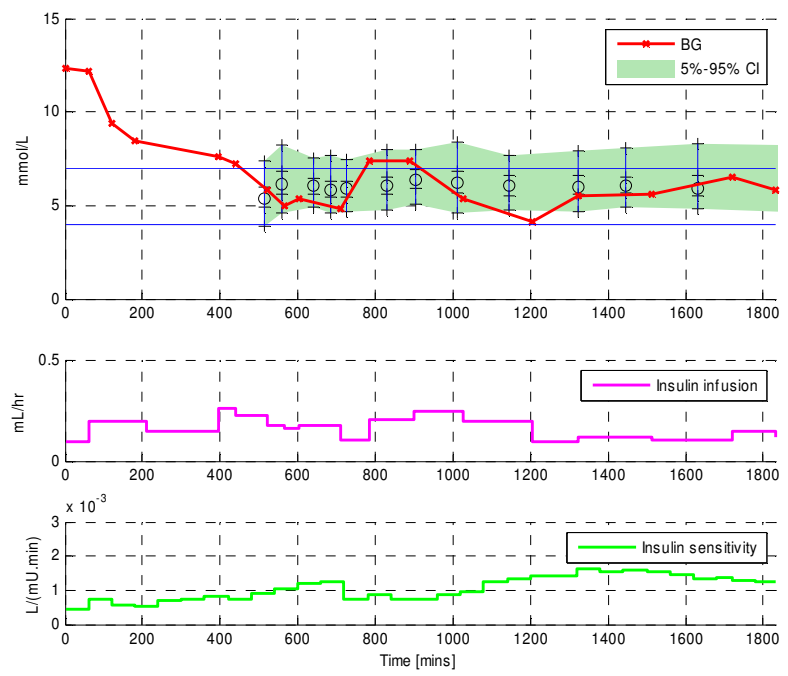

Fig. 7. NICU clinical control Trial \#1. The shaded area in the top panel represents BG forecast bounds, and the circle represents the target $\mathrm{BG}$ concentration.

\section{DISCUSSION}

Hyperglycaemia has been recently linked to poor outcomes for premature infants. However, there is currently no set protocol or best-practice method available. The success of model-based and model-derived control in limited trials in adult intensive care patients (Chase et al., 2007, Chase et al., 2008) suggest that model-based methods could be useful for metabolic management in neonates.

This study shows that time in a relevant glycaemic band is a clearer indication of control performance when comparing control protocols than a median value. The median blood glucose did not change significantly. However, time in band decreased dramatically in the presence of long measurement intervals out to 4 hours, indicating increased glycaemic variability and thus potentially worse outcomes (Egi et al., 2006). The BG IQR width reflected these changes in BG variability. However, time in band provides and easy-tovisualise method of comparison. Finally, cumulative distribution functions provide both a direct comparison and time in any desired glycaemic band.
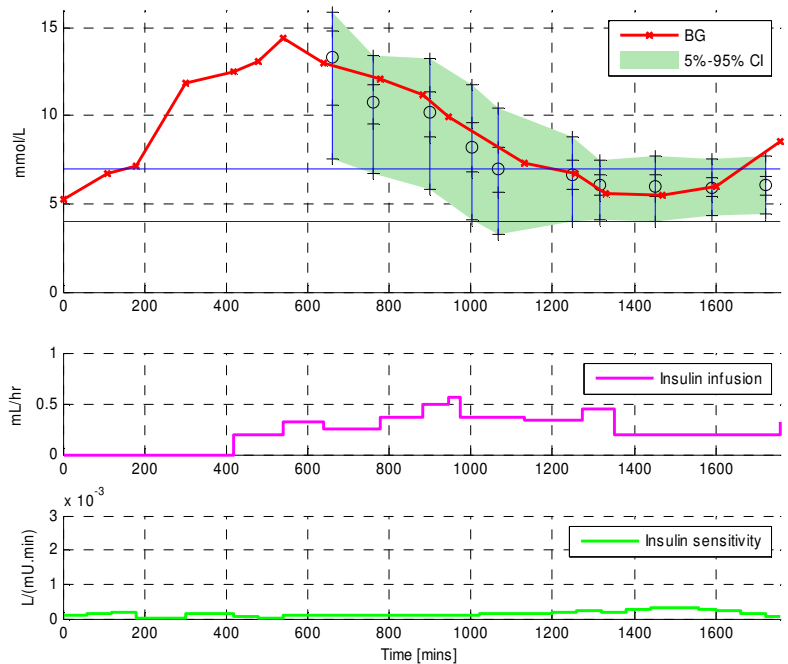

Fig. 8. NICU clinical control Trial \#2.

Pump flow rates in neonatal intensive care are very low given the very small doses. Pump accuracy can affect the quality of control, as well as insulin adsorption to pump tubing (Fuloria et al., 1998). This effect can be minimised by either adding albumin to the insulin mixture, or flushing the infusion pump tubing with insulin solution prior to use. Christchurch Women's hospital flushes all insulin tubing to minimise adsorption effects (Fuloria et al., 1998). Thus, this effect is not evident in the clinical data used.

The ideal range for blood glucose concentration in neonatal intensive care is under debate (Cowett and Farrag, 2004). Unlike adults, a major proportion of energy for brain metabolism is provided by fuels other than glucose (eg: ketones). Thus, the neonatal brain may be more resistant to hypoglycaemia compared to the adult. However, persistent low blood glucose concentration $(<2.6 \mathrm{mmol} / \mathrm{L})$ can reduce cerebral development and lead to long term neurological deficiencies (Lucas et al., 1988). For this study, the $4-7$ $\mathrm{mmol} / \mathrm{L}$ range was targeted, similar to several adult studies. However, to date, no outcome based study has provided a specific insight or result in this regard.

A paper-based protocol (SPRINT) developed from a similar model has been used on 394 adult intensive care patients (Chase et al., 2008). SPRINT uses 1-2 hourly measurements, and modulates both insulin and nutrition to achieve tight glycaemic control. It achieved $79 \%$ of measurements in a target $4-7 \mathrm{mmol} / \mathrm{L}$ band, which is similar to the $76 \%$ in these virtual trials within same band using a 2-hour measurement frequency. The added nutrition modulation provides another pathway for $\mathrm{BG}$ reduction that can be effective during periods of very low insulin sensitivity, 
particularly as adults appear to exhibit greater insulin effect saturation than preterm neonates. In contrast, extremely preterm infants lack substantial endogenous stores of energy, and thus must be fed constantly to maintain basal energy expenditure and provide excess for growth (Hume et al., 2005). Therefore, any similar system for neonates would likely be an "insulin-only" controller or one that sought to maximise nutritional inputs.

Further improvements to the model could incorporate daily nutritional and volume goals that can be set by clinicians with model-based targeted control taking care of glycaemia - thus relieving clinical staff from estimation and ad-hoc decision making. The ideal content and composition of nutritional regimes for preterm infants is still under debate. The proportions of dextrose, protein and lipids given in the NICU may be different to what an infant receives in-utero. Whilst the relevant major organs express many of the biological mechanisms responsible for glucose regulation from a relatively early age, the foetus depends upon the mother to control energy supply. Thus, the controller is essentially attempting to replicate some of the mother's functions, as well as account for the synchronised processes that regulate foetal growth that are perturbed by premature birth and life outside the womb.

\section{CONCLUSIONS}

A model of the neonatal glucose regulatory system is adapted from adult critical care. Simulated trials revealed the sensitivity of control performance to frequency of BG measurement. Optimised control schemes improved glycaemic control in simulation versus hospital control over a range of patients. Model and controller performance were validated in pilot clinical trials.

\section{REFERNCES}

Agus, M. S., Javid, P. J., Ryan, D. P. \& Jaksic, T. (2004) Intravenous insulin decreases protein breakdown in infants on extracorporeal membrane oxygenation. Journal of pediatric surgery, 39, 839-44; discussion 839-44.

Beardsall, K., Vanhaesebrouck, S., Ogilvy-Stuart, A. L., Vanhole, C., Palmer, C. R., van Weissenbruch, M., Midgley, P., Thompson, M., Thio, M., Cornette, L., Ossuetta, I., Iglesias, I., Theyskens, C., de Jong, M., Ahluwalia, J. S., de Zegher, F. \& Dunger, D. B. (2008) Early Insulin Therapy in Very-Low-BirthWeight Infants. N Engl J Med, 359, 1873-1884.

Cassady, G. (1966) Plasma volume studies in low birth weight infants. Pediatrics, 38, 1020-7.

Chase, J., Shaw, G. M., Wong, X. W., Lotz, T., Lin, J. \& Hann, C. E. (2006) Model-based glycaemic control in critical care - a review of the state of the possible. Biomedical Signal Processing and Control, 1, 3-21.

Chase, J. G., Shaw, G., Le Compte, A., Lonergan, T., Willacy, M., Wong, X.-W., Lin, J., Lotz, T., Lee, D. \& Hann, C. (2008) Implementation and evaluation of the SPRINT protocol for tight glycaemic control in critically ill patients: a clinical practice change. Critical Care, 12, R49.
Chase, J. G., Shaw, G. M., Lotz, T., LeCompte, A., Wong, J., Lin, J., Lonergan, T., Willacy, M. \& Hann, C. E. (2007) Model-based insulin and nutrition administration for tight glycaemic control in critical care. Curr Drug Deliv, 4, 283-96.

Chen, E. T., Nichols, J. H., Duh, S. H. \& Hortin, G. (2003) Performance evaluation of blood glucose monitoring devices. Diabetes Technol Ther, 5, 749-68.

Cowett, R. M. \& Farrag, H. M. (2004) Selected principles of perinatal-neonatal glucose metabolism. Semin Neonatol, 9, 37-47.

Ditzenberger, G. R., Collins, S. D. \& Binder, N. (1999) Continuous insulin intravenous infusion therapy for VLBW infants. J Perinat Neonatal Nurs, 13, 70-82.

Dweck, H. S. \& Cassady, G. (1974) Glucose Intolerance in Infants of Very Low Birth Weight. Pediatrics, 53, 189.

Egi, M., Bellomo, R., Stachowski, E., French, C. J. \& Hart, G. (2006) Variability of blood glucose concentration and short-term mortality in critically ill patients. Anesthesiology, 105, 244-52.

Ertl, T., Gyarmati, J., Gaal, V. \& Szabo, I. (2006) Relationship between hyperglycemia and retinopathy of prematurity in very low birth weight infants. Biology of the neonate, 89, 56-9.

Farrag, H. M., Nawrath, L. M., Healey, J. E., Dorcus, E. J., Rapoza, R. E., Oh, W. \& Cowett, R. M. (1997) Persistent glucose production and greater peripheral sensitivity to insulin in the neonate vs. the adult. Am J Physiol, 272, E86-93.

Fuloria, M., Friedberg, M. A., DuRant, R. H. \& Aschner, J. L. (1998) Effect of flow rate and insulin priming on the recovery of insulin from microbore infusion tubing. Pediatrics, 102, 1401-6.

Hann, C. E., Chase, J. G., Lin, J., Lotz, T., Doran, C. V. \& Shaw, G. M. (2005) Integral-based parameter identification for long-term dynamic verification of a glucose-insulin system model. Comput Methods Programs Biomed, 77, 259-270.

Hume, R., Burchell, A., Williams, F. L. \& Koh, D. K. (2005) Glucose homeostasis in the newborn. Early Hum Dev, 81, 95-101.

Kairamkonda, V. (2006) Does continuous insulin infusion improve glycaemic control and nutrition in hyperglycaemic very low birth weight infants? Arch Dis Child, 91, 76-79.

Lucas, A., Morley, R. \& Cole, T. J. (1988) Adverse neurodevelopmental outcome of moderate neonatal hypoglycaemia. Br Med J, 297, 1304-1308.

Natali, A., Gastaldelli, A., Camastra, S., Sironi, A. M., Toschi, E., Masoni, A., Ferrannini, E. \& Mari, A. (2000) Dose-response characteristics of insulin action on glucose metabolism: a non-steady-state approach. Am J Physiol Endocrinol Metab, 278, E794-801.

Van den Berghe, G., Wouters, P., Weekers, F., Verwaest, C., Bruyninckx, F., Schetz, M., Vlasselaers, D., Ferdinande, P., Lauwers, P. \& Bouillon, R. (2001) Intensive insulin therapy in the critically ill patients. N Engl J Med, 345, 1359-1367. 